\title{
Posture, 3D Real Body, Virtual Try-On: Toward fashion
}

\author{
Jean-Marc SURVILLE \\ Lectra, France
}

\begin{abstract}
Work on real bodies through the virtual try-on software give an important practice field. Students as well as skilled operators understand the real relationship between a garment and the body (1).

As an introduction a standard garment is mounted on a virtual mannequin.

Fitting is generally analyzed in a predicted result according to standard construction on standard mannequin. For our purpose, a common real person may wear a standard garment as well as garment constructed on her proper measurements. The virtual environment let the skill operator appreciate the fitting. In the case of a particular posture the 3D environment is a powerfully tool to understand the effects of the posture on the garment behavior.

The 3D body measurement software of body scanners is a precious tool for understanding the future garment dimension. A standard construction let appear the defaults of fitting between the garment and the body. Different successive approaches are presented to solve the different parts of the garment involved in the draping. The interactive process gives a theoretical and practical view of the fitting result before the real confirmation by sewing the real garment. In conclusion 3D real bodies issued from body scanners allow the introduction of fashion in real case studies thanks to 3D virtual try-on software.
\end{abstract}

Keywords: anthropometry, made to measure, virtual try-on

\section{Introduction}

The introduction of 3D virtual try-on software on the market reveals different interesting approaches from fitting, to virtual catwalks (11). The use of standard mannequins remains a common way to evaluate standard sizes. However statistics on the recent surveys give a more open view on the diversity of people morphologies.

The skill for garment fitting evaluation is based on tradition aesthetics (2).

The use of virtual mannequins having standard size volume drives the interest to the garment appearance.
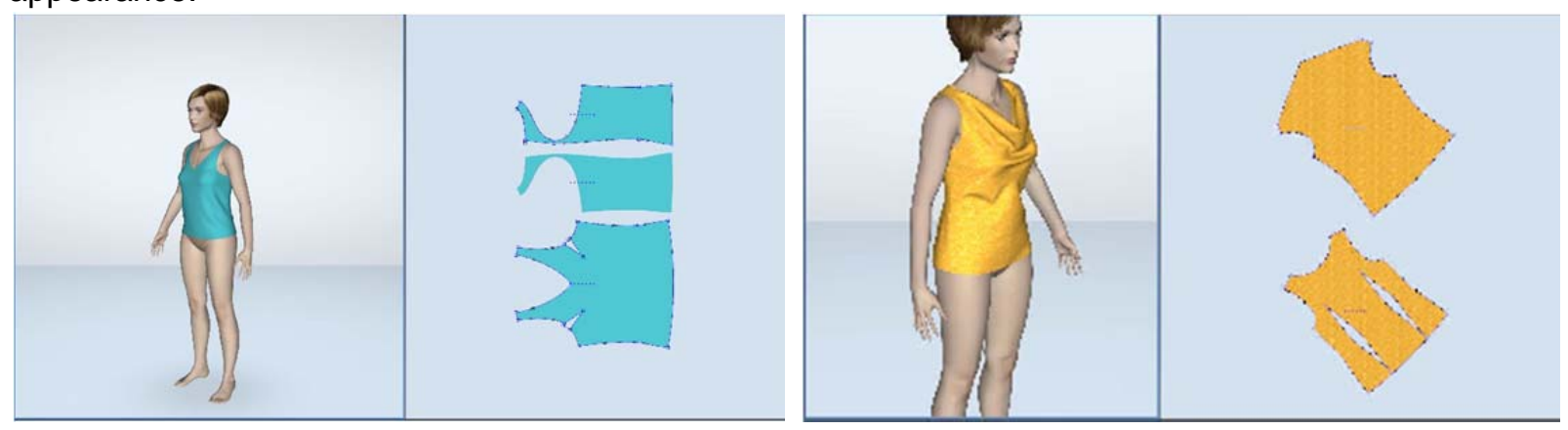

Views from the Lectra Modaris Solution

This process is dedicated to ready to wear thanks to a fashion process that enhances the appearance of the garment itself and pay few interest to the consumer.

\section{Real scanned student}

Body scanners offer different sets of measurements.

Some measurements are dedicated to classical anthropometry such as height, bust girth, waist girth, hip girth etc. 
Other measurements may be oriented to industrial interest such as ergonomics (10).

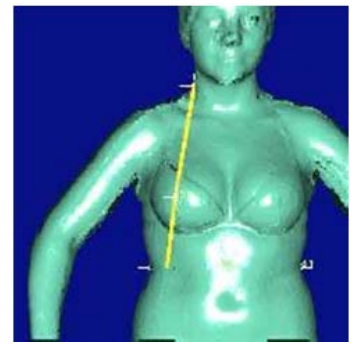

For the apparel industry, measurements may be dedicated to pattern construction. The neck to waist over bust is useful for dart applications.

\subsection{Preparing the try-on}
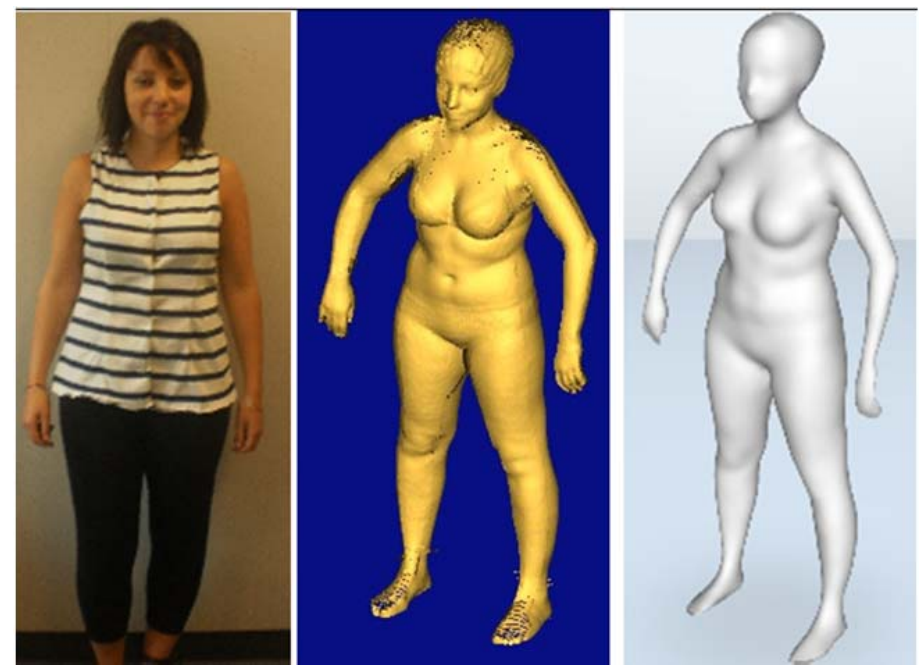

Figure 1 process to enter virtual world

The students have been scanned using a body scanner, and then introduced into the virtual world to process a virtual try-on on their own bodies

\subsection{Basic top body construction}

\subsubsection{How to flatten the bust?}

The students used a construction based on their measurements taken on their own scanned bodies. Ease was evaluated according to the style decided (1).

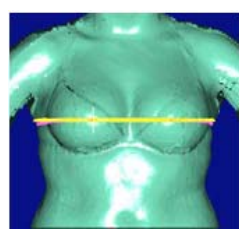

Bust girth

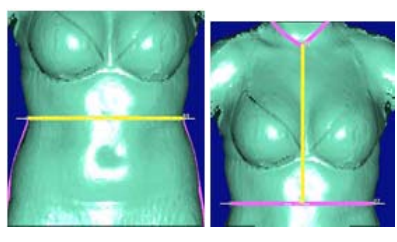

Waist front neck to $\mathrm{W}$

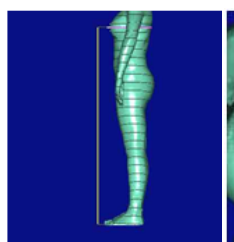

Bust heigh

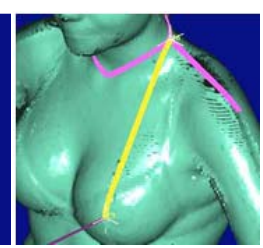

nipple to neck

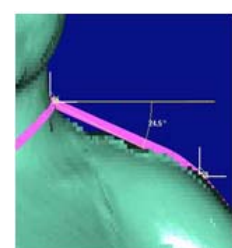

shoulder
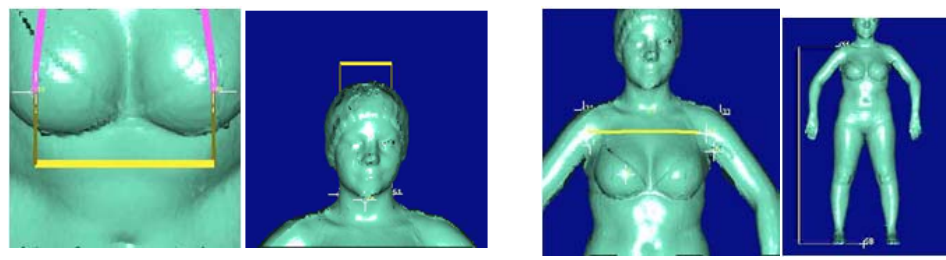

Nipples width, neck width, across front, and shoulder height.

We evaluate the silhouette front on side. This give the side part of the garment 
The above measurements are used to construct a flatten bloc at center front using neck to waist, neck width, and shoulder length and angle

On the side, waist, bust and shoulder line.

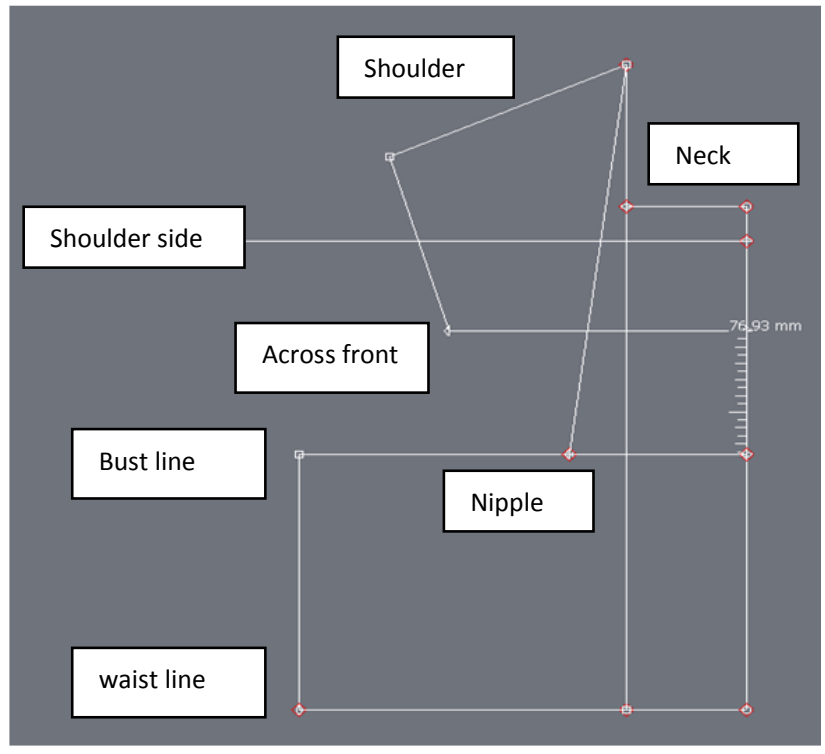

Figure 2 basic construction using body measurements

2.2.2. Flattening the $3^{\text {rd }}$ dimension.

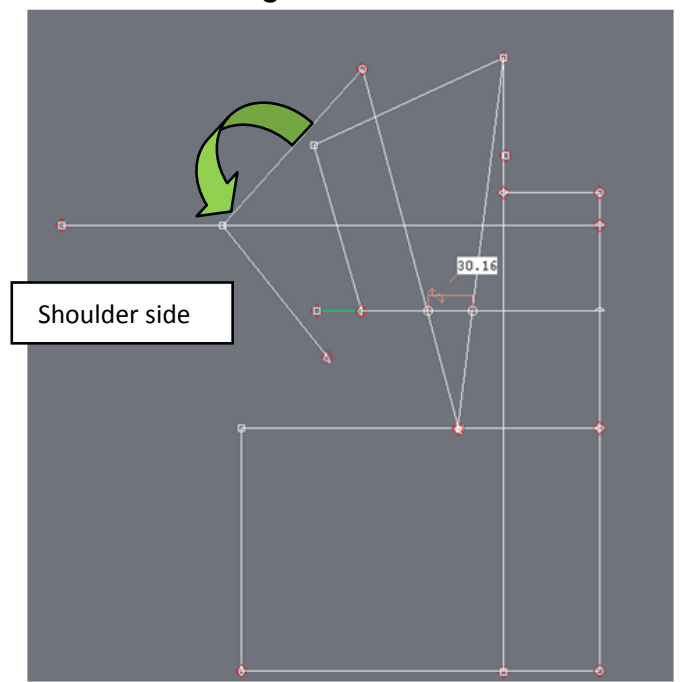

Construction using 2D pattern Lectra Modaris Software.
The line "nipple to neck" is used as the radius rotation to joint shoulder point to the shoulder line.

The dart is geometrically opened according to the personal dimensions. 


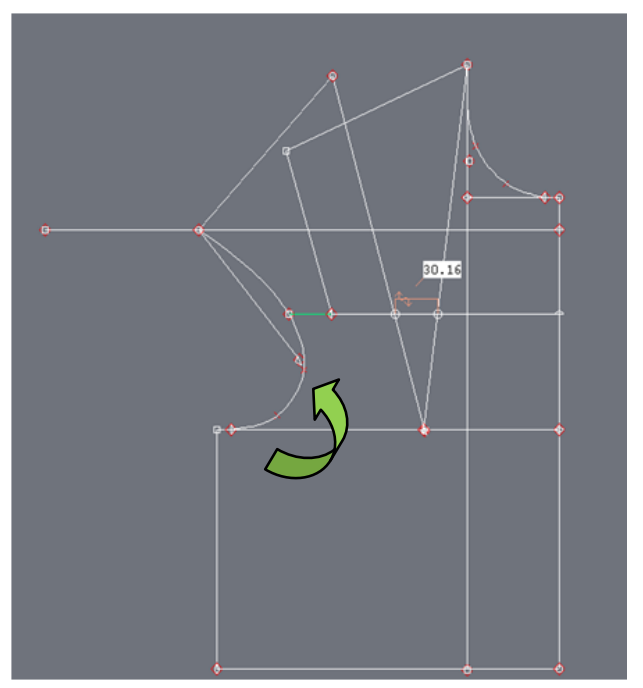

To finalize the armhole, adjustment on the across front is proceeded.

\subsubsection{Complete top construction}

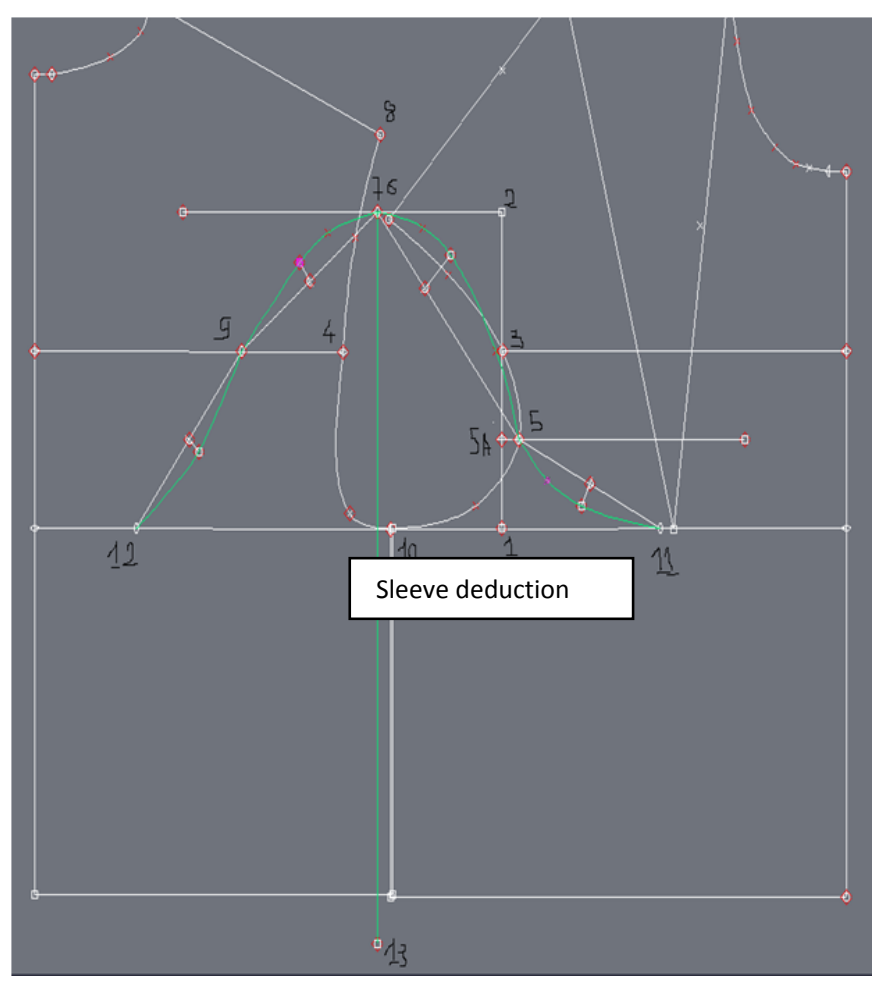

The back is constructed on the same principle.

The sleeve head is deducted from the total armhole. 


\subsection{Basic top body virtual try-on}

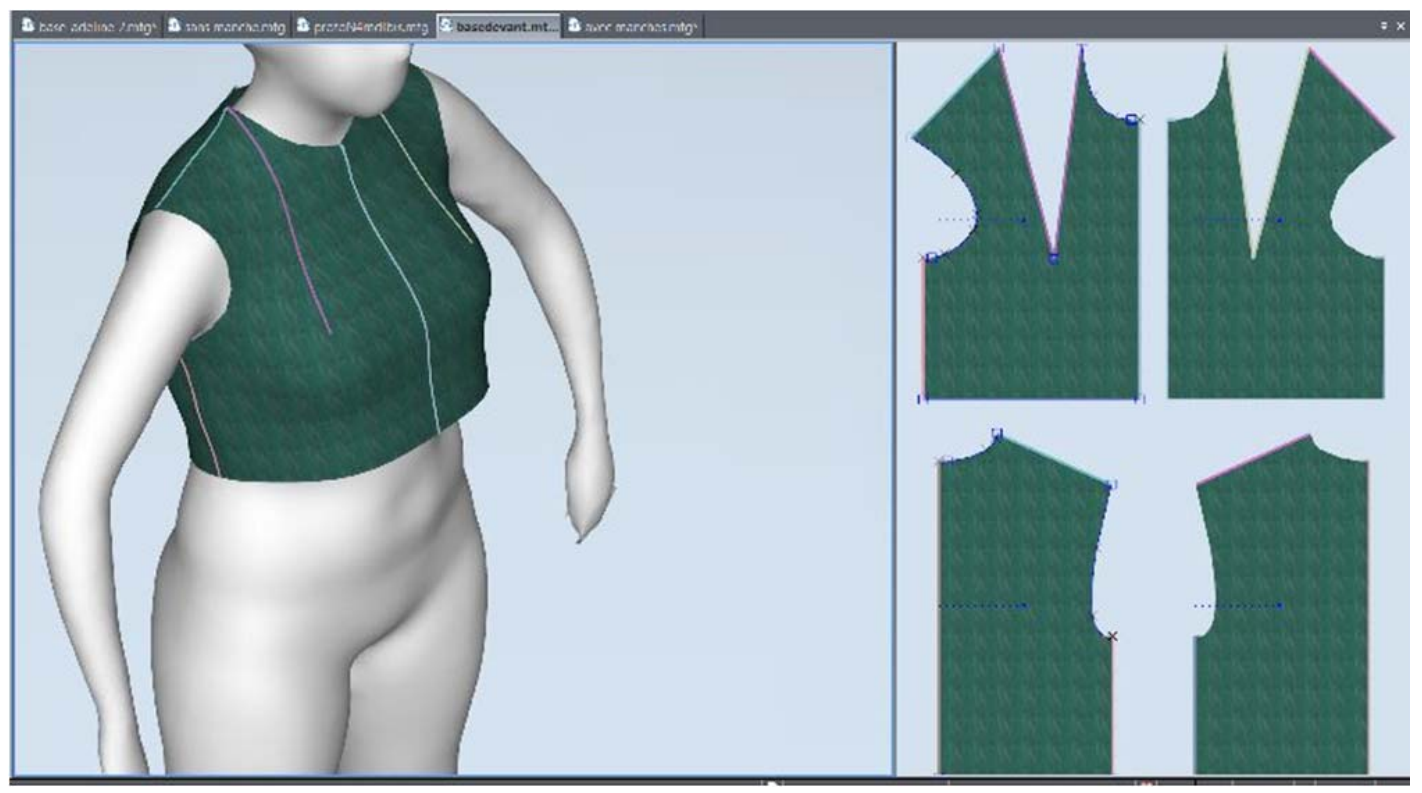

Fitting for balance front back may be adjusted directly in 3D On the block, pattern transformation may be applied according to skill.

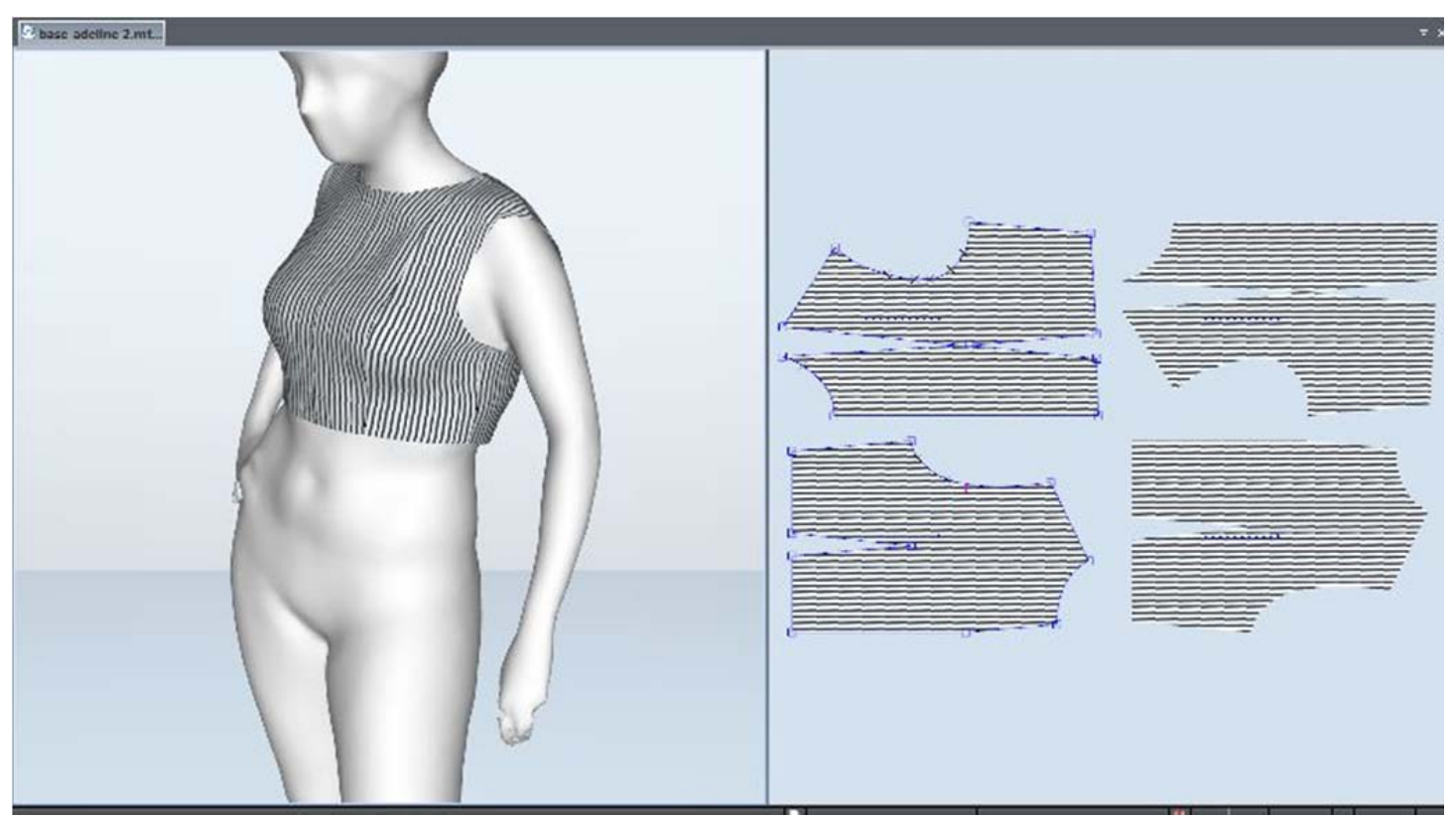

Fig 3 dart transformation on waist. 


\section{Test/Data Particular posture, 3D virtual try-on added value}

\subsection{The plus size sector may be treated as well}

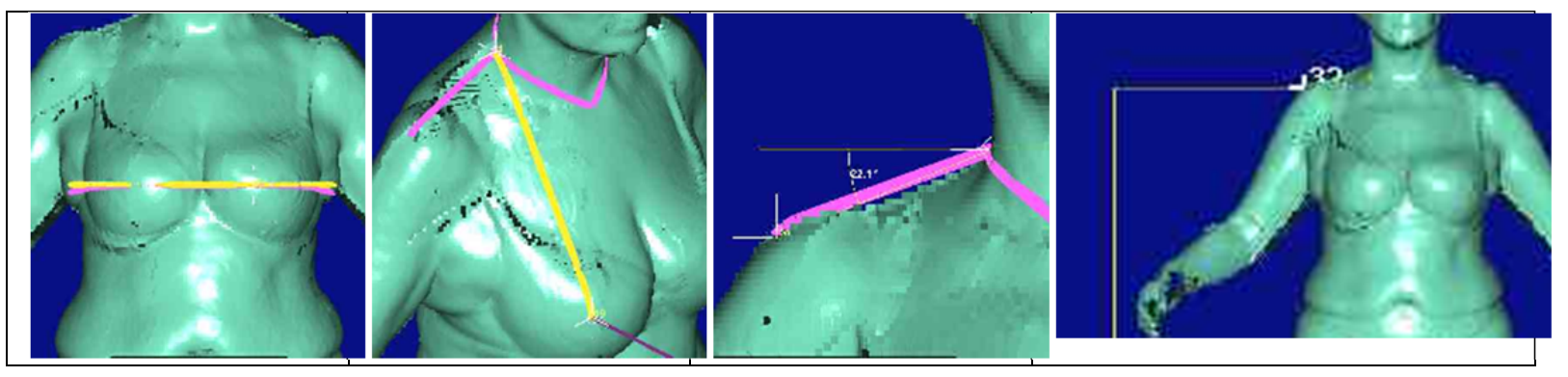

According to the process described, direct body measurements are used for the basic block construction.

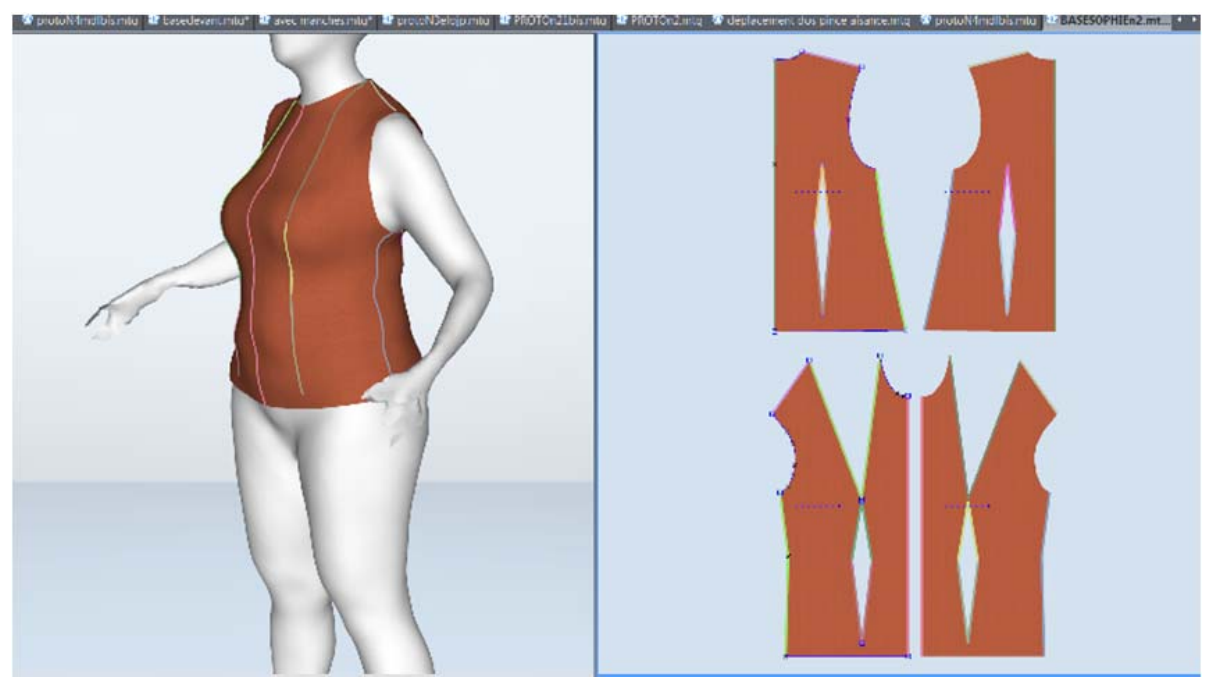

The top garment was virtually completed with sleeve, and controlled in 3D.

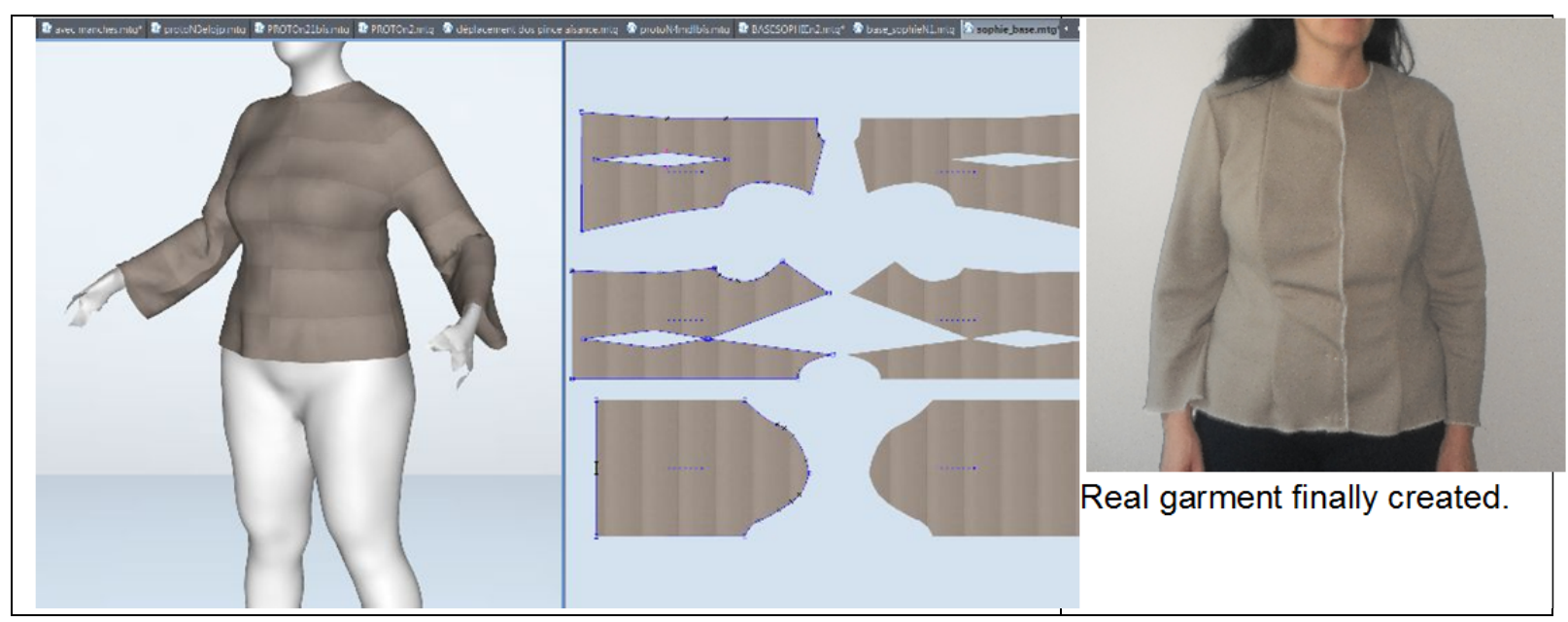




\subsection{Process on particular posture}

In case of a particular posture
the 3D environment is a
powerful tool to understand
the effects of the posture on
the garment behavior.
The 3D body measurement
software of body scanners is
a precious tool for
understanding the future
garment dimension.

Following the same process using body measurements the first prototype was virtually tried on.

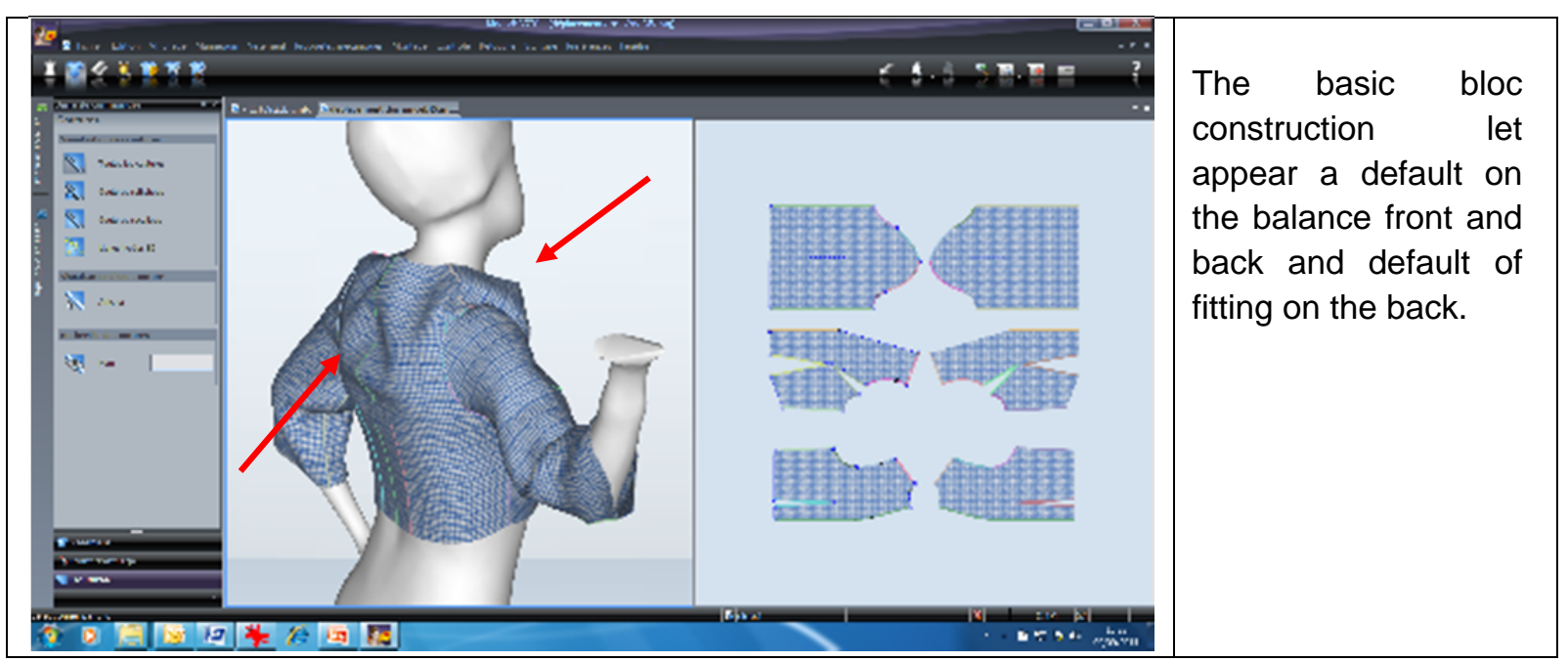

Fig. 4 Modaris 3D fit environment

The analysis of the defaults were based on "standards for fitting a basic garment" ((2) p 58)

Defaults appeared on the low back due to the
erect natural posture
The overlapped fabric may be reduced through
the back dart and immediately verified on the
try-on.




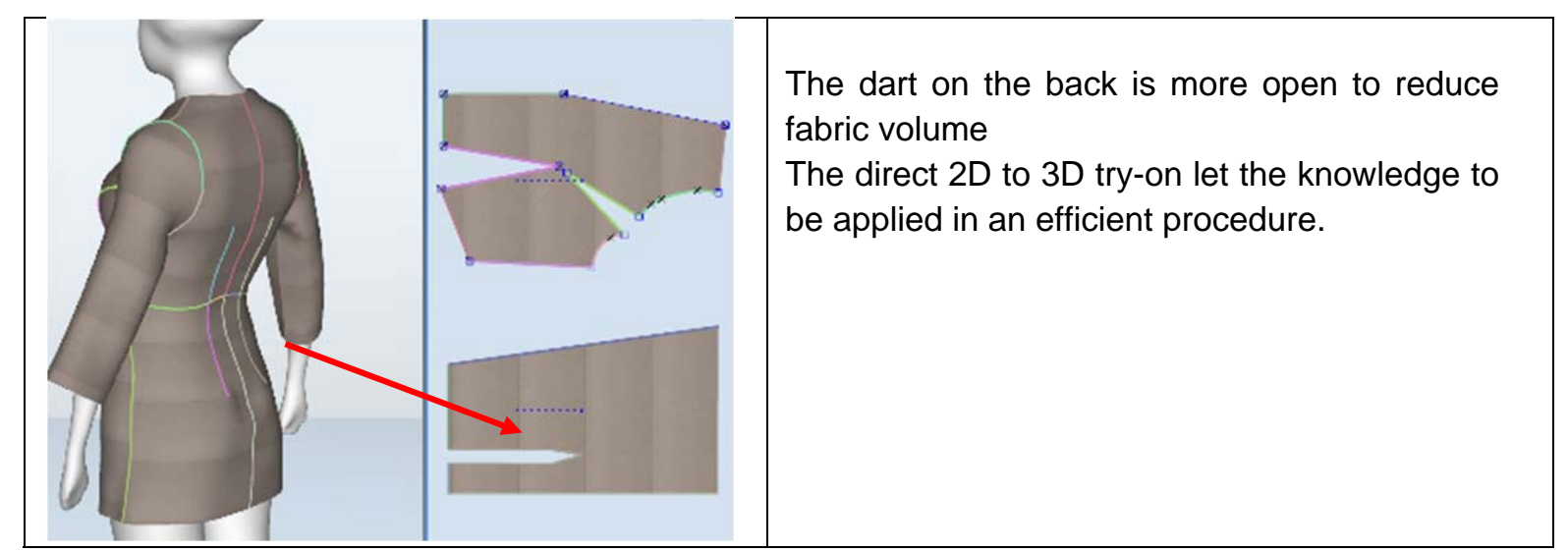

\section{The try-on revelation}

The construction was based on the real body, and has a different feeling than the approach proposed by some authors on the wooden classical mannequin (7).

The adjustment were not predicted but only adjusted according to fit analysis (2).

The value of a scanner in the basic bloc construction process as the provider of the 3D measurement is a key for the good fitting understanding.

\section{Discussion conclusion}

On this work, the virtual environment was used as a tool for understanding how measurements are involved in the 2D construction. The flattening was separated between center front and side. The dart was open thanks to the true body dimensions.

Few adjustments (including ease) were necessary to reach a good fitting.

We demonstrate here that a plus size candidate as well a particular posture student were treated.

The use of the 3D virtual try-on onto the true virtual bodies has an added value for the understanding of the interaction between body and garment.

This approach has been presented by some authors $(7,8)$. However our goal is the direct relationship between the body and the garment, analyzed in the real and virtual world and not only a realistic representation.

In an industrial context, the user becomes an efficient actor, with a minimum tool required to enhance creativity. Benefits may be estimated as saving the first prototypes and the fast cycle to simulation . The process may claim to refer to real human mannequin walking in defile.

As a matter of teaching, this session claims to let operators understand the reality of wearing a garment, in an alive context, far away from the workshop mannequins. 


\section{References}

(1) Jean-Marc SURVILLE, Apparel: From reality to virtual reality, International Conference on 3D Body Scanning Technologies, Lugano, Switzerland, 19-20 October 2010 p 89

(2) Elizabeth L. Liechty, Della N. Pottberg-Steineckert, Judith A. Rasband, Fitting and Pattern Alteration: A Multi-Method Approach. 1992 New York Fairchild publications

(3) Catherine Black, Modaris, Diamino, and JustPrint for Apparel Design, . 2007 New York Fairchild publications

(4) Xiaoqun Dai, Takao Furukawa, Shigeru Mitsui, Masayuki Takatera, Yoshio Shimizu, (2001) "Drape formation based on geometric constraints and its application to skirt modelling", International Journal of Clothing Science and Technology, Vol. 13 Iss: 1, pp.23 - 37

(5) Susan Ashdown, Suzanne Loker, co-leaders (Cornell); Margaret Rucker (UC Davis) Improved Apparel Sizing: Fit and Anthropometric 3D Scan Data NTC Project: S04-CR01 http://www.ntcresearch.org/projectapp/?project=S04-CR01

(6) M. Wacker and Al., Simulation and Visualisation of Virtual Textiles for Virtual Try-On Research Journal of Textile and Apparel Vol. 9 No. 12005

(7) Choong Hyo Kim, In Hwan Sul, Chang Kyu Park, Sungmin Kim, (2010) "Automatic basic garment pattern generation using three-dimensional measurements", International Journal of Clothing Science and Technology, Vol. 22 Iss: 2/3, pp.101 - 113

(8) Young Sook Cho, Keiichi Tsuchiya, Masayuki Takatera, Shigeru Inui, Hyejun Park, Yoshio Shimizu, (2010) "Computerized pattern making focus on fitting to 3D human body shapes", International Journal of Clothing Science and Technology, Vol. 22 Iss: 1, pp.16 - 24

(9) Elizabeth Bye, Ellen McKinney, (2010) "Fit analysis using live and 3D scan models", International Journal of Clothing Science and Technology, Vol. 22 Iss: 2/3, pp.88-100

(10) Pheasant, S. Bodyspace : Anthropometry, Ergonomics and the Design of the Work, Taylor \& Francis, 1996

(11) Ran MACHTINGER Perfect Garment Fitting Using 3D Body Scanning, International Conference on 3D Body Scanning Technologies, Lugano, Switzerland, 19-20 October 2010 p 59 